\title{
Lidil
}

Revue de linguistique et de didactique des langues

47 | 2013

Le verbe pour exprimer le temps

\section{L'argumentation en français scientifique à un niveau A2 du Cadre européen commun de référence pour les langues}

Françoise Olmo Cazevieille

\section{OpenEdition}

Journals

Édition électronique

URL : http://journals.openedition.org/lidil/3274

DOI : $10.4000 /$ lidil.3274

ISSN : 1960-6052

Éditeur

UGA Éditions/Université Grenoble Alpes

\section{Édition imprimée}

Date de publication : 31 mai 2013

Pagination : 149-168

ISBN : 978-2-84310-247-9

ISSN : $1146-6480$

Référence électronique

Françoise Olmo Cazevieille, "L'argumentation en français scientifique à un niveau A2 du Cadre européen commun de référence pour les langues », Lidil [En ligne], 47 | 2013, mis en ligne le 01 décembre 2014, consulté le 30 avril 2019. URL : http://journals.openedition.org/lidil/3274; DOI : 10.4000/ lidil.3274 


\title{
L'argumentation en français scientifique à un niveau $\mathrm{A} 2$ du Cadre européen commun de référence pour les langues
}

\author{
Françoise Olmo Cazevieille*
}

\begin{abstract}
RÉSUMÉ
L'argumentation est une opération énonciative par laquelle un locuteur essaie d'influencer son interlocuteur dans une décision, un point de vue, etc. Dans ce sens, elle suppose une interaction entre au moins deux personnes. Cette opération complexe semble n'être envisageable qu'à des niveaux consolidés d'apprentissage de la langue française scientifique. Cependant, je vais montrer, en m'appuyant sur l'activité du débat, qu'elle peut être appliquée à des niveaux plus élémentaires. Grâce à cette technique, les étudiants apprendront à argumenter sur des sujets spécialisés.
\end{abstract}

\begin{abstract}
Argumentation is an enunciative operation by which a speaker is trying to influence his interlocutor with a decision, a point of view, etc. In this sense, it implies an interaction between at least two people. This complex operation seems to be possible only at an advanced level of learning in French scientific language. However, based on debate activity, I will demonstrate that argumentation may occur on more elementary levels of learning. This technique proves efficient for students to learn how to argue on specialized topics.
\end{abstract}

«L'important n'est pas de convaincre, mais de donner à réfléchir.»

Bernard WERBER, Le père de nos pères.

* Universitat Politècnica de València, GALE (Grupo de Análisis de Lenguas de Especialidad). 


\section{Introduction}

Le contexte institutionnel dans lequel je me trouve à l'Université polytechnique de Valence (UPV) en Espagne, me contraint à enseigner le français à des niveaux élémentaires. En effet, très peu d'étudiants arrivent dans l'enseignement supérieur avec des connaissances en langue française et par conséquent, la constitution de groupes de niveau intermédiaire ou avancé est assez rare. Par ailleurs, je me vois obligée d'adapter les contenus aux différents besoins académiques et professionnels des étudiants et donc de leur proposer, autant que faire se peut, des activités axées sur leur cursus scientifique. Cette conjoncture exigeante me mène à développer des activités didactiques dans lesquelles l'argumentation trouve toute sa place. Et, bien que celle-ci soit une opération complexe qui souvent n'est envisagée qu'à des niveaux consolidés d'apprentissage de la langue française scientifique, je vais montrer ici qu'elle peut être réalisée à des niveaux plus élémentaires. Après avoir examiné les bases théoriques et méthodologiques qui sous-tendent mon approche, je m'attacherai à décrire, dans une démarche actionnelle (Conseil de l'Europe, 2000, p. 15), l'activité du débat conçue de façon à aider les apprenants à développer leur compétence communicative en argumentant sur des sujets spécialisés qui les motivent. L'analyse des résultats de cette technique pédagogique me permettra d'atteindre ma finalité.

\section{Bases théoriques}

Avant de me référer à l'argumentation aux niveaux élémentaires de français, j'aimerais clarifier brièvement pourquoi j'ai choisi dans mon titre la dénomination de français scientifique, et ce, afin de situer le type de français que j'enseigne à l'heure actuelle à l'UPV. Le contexte, l'Espagne, le place à l'intérieur du français langue étrangère, l'institution, l'université, dans la catégorie du français de spécialité que nous entendons à l'instar de Mangiante et de Parpette (2004) comme «une approche globale d'une discipline ou d'une branche professionnelle, ouverte à un public le plus large possible» (p. 17). Dispenser les cours uniquement dans des écoles d'ingénieurs m'a fait opter pour une dénomination moins ample que cette dernière, celle de français scientifique. Bien qu'à certains moments donnés, le français enseigné ait fait partie du Français sur Objectifs Spécifiques (FOS) par «souci de l'adapter à des publics adultes souhaitant acquérir ou perfectionner des com- 
pétences en français pour une activité professionnelle ou des études supérieures» (Cuq, 2003, p. 109), aujourd'hui, le profil des étudiants beaucoup plus hétérogène (venant de cursus très différents), m'empêche de cibler les contenus aux différentes spécialisations ${ }^{1}$ d'une seule ingénierie. Je prendrai pour étayer mes propos l'exemple du groupe de français de niveau A2 de l'école supérieure de télécommunication dans lequel j'ai enseigné au deuxième semestre de l'année scolaire 20112012 et dont nous allons examiner les écrits ci-après. Ce groupe était formé, outre quelques étudiants de cette école, par des apprenants venant d'aéronautique, d'architecture et de communication audiovisuelle. De ce fait, les activités professionnelles en présence dans le groupe classe étaient très variées. Le choix de cette dénomination, le français scientifique $^{2}$, vient donc de la situation communicative dans laquelle je me trouve, mais repose aussi sur la méthodologie suivie pour enseigner la langue française. Avant de passer à la description de celle-ci, arrêtons-nous un instant sur cette situation communicative et ses implications dans l'enseignement du français langue étrangère. Le français scientifique appartient au français de spécialité; leurs traits définitoires coïncident :

- la langue de spécialité n'est en aucune façon une autre langue;

- la langue de spécialité rend compte des connaissances du domaine étudié;

- «la sémantique de la langue de spécialité et de sa terminologie est un domaine essentiel de son étude» (Kocourek, 1991, p. 41);

- les critères pour l'analyser peuvent être les mêmes que ceux utilisés dans la langue générale (Olmo, 2008, p. 198).

Cependant, les domaines de spécialité englobent tous les domaines dans lesquels les connaissances sont spécialisées c'est-à-dire, toutes les situations de communication professionnelles. À la manière de Bédard (1986, p. 101-111), je différencie dans les domaines de spécialité les réalités humaines (droit, finance, administration, etc.) des réalités non humaines, les sciences dites exactes (géologie, chimie, biologie, etc.) et l'ensemble des techniques. C'est pourquoi je me situe dans le français

1. Les ingénieurs en télécommunications, par exemple, peuvent choisir une des quatre branches suivantes : spécialiste en systèmes de télécommunications, en systèmes électroniques, en téléinformatique ou en son et image.

2. Réduction de français scientifique et technique. 
scientifique. En outre, ce sont les textes produits par des spécialistes d'un domaine scientifique qui vont me servir de base pour réaliser les activités argumentatives. Ces textes ne présentent pas tous le même niveau de spécialité. Celui-ci varie selon, entre autres, les destinataires. Dans ce sens, Cabré explique :

La pertinence d'un texte à un niveau haut, moyen ou bas se détermine par les caractéristiques des destinataires, son support et ses finalités. Ainsi, un texte produit par un spécialiste pour des étudiants peut se définir comme de niveau moyen. (Cabré, 2007-2008, p. 40)

L'utilisation de ce type de textes dans l'élaboration d'activités complémentaires à un manuel de français langue étrangère général, permet à l'enseignant d'un public scientifique de traiter non seulement des sujets spécialisés, mais aussi de les mettre en contact avec des textes authentiques, réalisés par des experts français pour un public français. La réalisation de l'exercice implique auparavant, comme nous le verrons ci-après, la compréhension des textes : lecture, compréhension du message, réexpression. La nature des textes scientifiques ajoute à cette démarche un bagage cognitif spécialisé que l'étudiant doit acquérir, intégrer et relier à ses connaissances en langue maternelle pour réaliser l'activité demandée. En effet, en lisant la documentation, il apprend la terminologie du domaine et son emploi. Il se familiarise avec la langue de spécialité.

Par ailleurs, la méthodologie choisie se fonde d'une part, sur les orientations du Cadre européen commun de référence pour les langues (désormais CECR ou Cadre) et d'autre part, sur les pratiques scientifiques suivies en France en contextes scolaires comme nous le verrons infra. L'approche de type actionnel retenue par le Cadre, considère :

$[\ldots]$ avant tout l'usager et l'apprenant d'une langue comme des acteurs sociaux ayant à accomplir des tâches (qui ne sont pas seulement langagières) dans des circonstances et un environnement donnés, à l'intérieur d'un domaine d'action particulier. [...] Il y a «tâche» dans la mesure où l'action est le fait d'un (ou de plusieurs) sujet(s) qui y mobilise(nt) stratégiquement les compétences dont il(s) dispose(nt) en vue de parvenir à un résultat déterminé. La perspective actionnelle prend donc aussi en compte les ressources cognitives, affectives, volitives et l'ensemble des capacités que possède et met en œuvre l'acteur social. (Conseil de l'Europe, 2001, p. 15)

Il ressort de cet extrait que la «tâche» peut être langagière, mais peut aussi ne pas l'être et qu'elle est indissociable de l'action. En résumé et 
à mon avis, la tâche correspond à toute activité d'apprentissage qu'elle soit de découverte, de systématisation ou d'utilisation dans laquelle l'apprenant joue un rôle actif. De nombreuses techniques pédagogiques placent l'apprenant au centre de l'apprentissage, à savoir : les jeux de rôle, les simulations, les réunions, l'expérimentation, la conduite de projets, etc. Pour cette étude, comme je l'avais annoncé dans l'introduction, je me centrerai sur le débat comme exemple de pratiques actives développant le discours argumenté. Cette activité permet de renforcer aussi, nous y reviendrons plus loin, l'autonomisation, c'est-à-dire «l'acquisition progressive de la capacité d'apprendre» (Cuq, 2003, p. 22), la créativité et la compétence de communication en langue française des apprenants. L'approche actionnelle qui tient bien sûr aussi compte de l'approche communicative, en se basant sur l'action, adopte une direction cognitive de l'apprentissage établie sur le constructivisme (Ausubel, 1968) et le socioconstructivisme (Bruner, 1960 ; Vygotsky, 1978). Pour les constructivistes, l'enseignement-apprentissage part du fait que chacun se construit à partir des connaissances antérieures et des expériences vécues; pour les socioconstructivistes, cet apprentissage doit se faire en interaction avec les pairs et l'enseignant. Le cognitivisme se centre sur les apprenants et les place au cœur de l'action. Il s'intéresse au processus mental suivi par chacun d'eux lors de l'apprentissage. Ces allers-retours entre action et raisonnement m'amènent à me référer aussi et ce, pour justifier mon choix pédagogique, aux nombreux auteurs qui se sont intéressés au décloisonnement des disciplines en faveur d'une formation plus humaniste. Ainsi, Edgar Morin, dans les années 1950, se réfère dans ce sens aux découvertes réalisées par des chercheurs ayant une vision systémique tels que Charles Darwin (théorie de l'évolution) ou Alfred Wegener (théorie de la dérive des continents), il observe :

Les disciplines sont pleinement justifiées intellectuellement à condition qu'elles gardent un champ de vision qui reconnaisse et conçoive l'existence des liaisons de solidarités. Plus encore, elles ne sont pleinement justifiées que si elles n'occultent pas de réalités globales. (Morin, 2003, p. 1-2)

\section{Genelot rapporte :}

«Une fureur sacrée de comprendre pour faire et de faire pour comprendre, qui passe toute philosophie», voici ce que Paul Valéry percevait chez Léonard de Vinci qui ne se contentait pas «de penser pour faire», mais était aussi continuellement engagé dans le «faire pour penser». (Genelot, 2011, p. 1) 
Je m'intéresse donc, tout d'abord, à l'argumentation par son lien avec la connaissance, car comme l'exprime Plantin :

L'argumentation a une face cognitive : argumenter c'est exercer une pensée juste. Par une démarche analytique et synthétique, on structure un matériau; puis, on met en examen un problème, on réfléchit, on explique, on démontre, au moyen d'arguments, de raisons, de preuves. On fournit des causes. La conclusion de l'argumentation est une découverte, elle produit de l'innovation, ou rien moins que de la connaissance. (Plantin, 1996, p. 15)

Parmi les différentes théories de l'argumentation qui offrent un panorama à la fois contrasté et complémentaire, j'entends ici, l'argumentation dans l'acception classique de Grize (1990), c'est-à-dire «fournir des arguments, des raisons à l'appui ou à l'encontre d'une thèse. Ainsi argumenter renvoie à justifier, expliquer, étayer» (p. 40). Dans ce sens, «l'argumentation considère l'interlocuteur, non comme un objet à manipuler mais comme un alter ego auquel il s'agira de faire partager sa vision » (Grize, 1990, p. 40). Le choix du débat comme activité pédagogique m'amène à considérer, comme Dolz et Schneuwly (1998), le débat et le fait d'argumenter comme synonymes. Pour ces auteurs, «le débat est conçu comme un outil de construction collective d'une solution, régulé par un modérateur qui facilite la dynamique des échanges » (p. 165). À mon avis, le débat permet de mettre en lumière différentes positions et le fait de devoir argumenter mène quelquefois à un changement des représentations totales ou partielles de la position des personnes qui y participent.

Ayant affaire à un public de cursus scientifique et soucieuse d'offrir des activités en relation avec leurs intérêts académiques et professionnels, mon regard s'est porté sur les travaux des didacticiens s'intéressant à l'argumentation en classe de sciences. De nombreuses études ont été menées en didactique des sciences depuis une vingtaine d'années. Des auteurs tels Joshua et Dupin (1989) étudient la construction des connaissances scientifiques grâce au débat en classe où interactions langagières et argumentation se combinent. Dans le contexte scolaire en France, des travaux ont aussi été effectués : ceux de Douaire (2004); ceux qui ont été recueillis dans la revue Aster consacrée aux recherches en didactique des sciences expérimentales, notamment dans le numéro 38 (Jacobi \& Peterfalvi, 2004) sur les interactions langagières; ceux de la revue Pratiques dont je citerai en exemple, le numéro 96 (1997) ou encore le livre de Buty \& Plantin (2008). 
Mon étude se situe dans la lignée des travaux de Simonneaux \& Simmoneaux (2005) et Albe (2006) qui s'intéressent à l'enseignement des questions socioscientifiques controversées, mouvement éducatif intitulé Sciences-Technologies-Société (STS) qui a émergé dans les années 1970, en particulier en Angleterre et en Amérique du Nord. Ce mouvement rejoint le décloisonnement des disciplines que je viens de mentionner, car «il considère que les sciences et les technologies quoique différentes interagissent entre elles et avec la société» (Simmoneaux \& Albe, 2008, p. 118). En France, on parle de «questions socialement vives (QSV)» (Legardez \& Simonneaux, 2006), car elles suscitent des débats en relation avec les représentations sociales, le système des valeurs, entre spécialistes dans les domaines professionnels, etc. Les questions socialement vives m'intéressent parce qu'elles mobilisent différents domaines de référence et «favorisent chez les élèves la compréhension que la science est en construction, provisoire, que les modèles ne représentent pas le réel, que la science est porteuse de valeurs, qu'elle est inséparable de la société » (Simmoneaux \& Albe, 2008, p. 120).

Par conséquent, le cadre théorique de ma méthodologie s'appuie sur une approche actionnelle de direction cognitive dans laquelle j'utilise l'argumentation comme une technique dans le but de combiner les connaissances de la langue scientifique et de la langue générale et par là-même, je développe les compétences transversales (pour former des citoyens) qui automatiquement en découlent. Les questions socialement vives traitées sous forme de débat argumenté impliquent un niveau de langue consolidé et m'amène au vif du sujet : est-il possible d'argumenter à un niveau A2?

\section{Bases méthodologiques}

L'argumentation est présente à tous les niveaux d'apprentissage. Certains auteurs comme Golder (1996) qui étudient le développement cognitif de l'enfant ont observé que l'argumentation se manifeste dès l'âge de 2 ans au travers de la justification. Cette auteure ajoute, qu'elle «est maîtrisée dès le plus jeune âge dans des situations "naturelles" où la négociation sociale constitue une véritable raison d'argumenter» (Golder, 1996, p. 123). Selon la psychologie du développement, l'argumentation se complexifie avec l'âge et pour cette même auteure, les compétences argumentatives peuvent être optimum vers 16-17 ans. Ce 
qui veut dire que les apprenants adultes devraient être formés à l'argumentation dans leur langue maternelle.

Par ailleurs, selon le programme des langues vivantes français du ministère de l'Éducation nationale, le deuxième niveau de compétence fixé pour toute l'Europe doit être atteint en fin de troisième et selon le programme des quatrièmes et des troisièmes ${ }^{3}$ : «Dans leurs deux langues vivantes, les élèves apprennent à expliquer, argumenter, voire débattre. Ils enrichissent aussi leur connaissance des cultures étudiées.» On peut donc dire que l'argumentation est présente à tous les niveaux d'enseignement en Europe et qu'elle peut être intégrée dans l'apprentissage de la langue cible :

Non pas comme l'acquisition d'une capacité ${ }^{4}$ nouvelle mais comme le transfert de cette capacité déjà acquise dans un autre moyen d'expression. Si l'opération réussit, l'apprenant pourra étendre son registre de capacités de communication dans la langue étrangère sans référence à la langue maternelle. (Widdowson, 1991, p. 87-88)

Il est intéressant de retenir qu'à un niveau A2, les élèves apprennent à expliquer, argumenter, voire débattre, car ceci signifie que l'initiation à l'argumentation, au débat, doit être menée dans les niveaux élémentaires afin que les étudiants puissent être capables d'argumenter en autonomie au niveau suivant.

\section{Organisation et description de l'activité}

\subsection{Le débat}

Le débat est défini par le Trésor de la langue française informatisé ${ }^{5}$ comme «action de débattre; discussion généralement animée entre interlocuteurs exposant souvent des idées opposées sur un sujet donné». Il est souvent source de controverse, d'altercation. Abordé à la fin du niveau A2, il engendre simplement des discussions et se veut collaboratif. En effet, les apprenants doivent travailler en tandem et arriver

3. Ministère de l'Éducation nationale. (2012). Repéré à www.education.gouv. $\mathrm{fr} / \mathrm{cid} 81 /$ les-programmes.html

4. Nous avons adapté la citation d'origine à l'argumentation en modifiant le pluriel de «capacités » au singulier.

5. TFLI. (2012). Repéré à http://atilf.atilf.fr/dendien/scripts/tlfiv5/visusel.exe? $11 ; \mathrm{s}=2724057900 ; \mathrm{r}=1 ;$ nat $=; \mathrm{sol}=0$ 
à une prise de position commune sur le sujet traité. Il est employé comme une passerelle qui va permettre aux apprenants de mobiliser leurs acquis (les connaissances vues en A2) et de développer leur compétence à communiquer langagièrement afin de les préparer au niveau suivant sur l'échelle du Cadre, le B1.

À un niveau élémentaire, l'activité du débat argumenté doit, à mon avis, être menée par étapes. En effet, il convient d'accompagner chaque étudiant pour qu'il construise pas à pas son argumentaire. Lors de l'acquisition des connaissances argumentatives sur le sujet, l'enseignant fournira un maximum de supports (articles choisis, vidéos et autres supports iconiques), de ressources (pages web de dictionnaire, d'orthographe, de conjugaison) et surtout sera disponible pour répondre de façon individuelle ou collective, aux questions qui surgiront dans la classe. Ainsi les blocages de type cognitif et linguistique immédiatement résolus et expliqués seront évités. De sorte que, dans un premier temps, le débat n'est travaillé qu'en binômes. En effet, je pense que la réalisation de la tâche en petits groupes, deux ou trois maximum selon le nombre d'étudiants, favorise la prise de parole de tous les pairs. Plus détendus, les étudiants se mettent à parler sans inhibition et l'interaction devient naturelle. Ce succès dans la participation serait difficile à obtenir si l'activité était proposée au groupe classe au complet car souvent, ce sont toujours les plus extravertis ou les meilleurs qui participent le plus.

\subsection{La méthodologie}

Aujourd'hui, il n'existe aucune méthode de français scientifique conçue pour un public de niveau élémentaire, car celui-ci est peu nombreux et très spécifique. En outre, certains chercheurs pensent que la spécialité ne doit être traitée qu'à partir d'un certain niveau d'apprentissage (Mangiante \& Parpette, 2004). Pour pouvoir satisfaire les besoins de mes étudiants qui étudient le français à des fins académiques et professionnelles, je suis contrainte d'organiser les unités en deux moments : tout d'abord, l'utilisation d'une méthode de français général qui me permet de traiter des situations de la vie quotidienne dans lesquelles les étudiants peuvent se retrouver lors d'un séjour en France ou dans un pays francophone, puis, une partie plus scientifique et technique où je traite des thèmes de la spécialité concernée basés sur la découverte de documents techniques (Olmo, 2008, p. 201-205) ou de débat argumenté comme c'est le cas ici, afin de faire évoluer les apprenants en 
situation de communication scientifique. La méthode générale suivie avec ce groupe est Latitudes, niveau 1 de chez Didier. Ce manuel est composé de douze unités : les six premières couvrent le niveau A1 et les six suivantes, le A2. La première partie est vue dans le module ou unité d'enseignement du premier semestre, la seconde au deuxième. Deux unités sont spécialement en rapport avec le débat, l'unité 6 qui est focalisée sur l'expression de point de vue et l'unité 11 qui développe l'expression de l'accord et du désaccord. Le débat argumenté est développé après cette dernière unité.

\subsection{Le profil des étudiants}

Les étudiants de ce groupe de français scientifique dispensé à l'école supérieure de télécommunication viennent, comme je l'ai signalé supra, de cursus différents, la moyenne d'âge est environ d'une vingtaine d'années, l'âge le plus fréquent étant 19 ans car cette unité d'enseignement est située en première année universitaire. Il faut noter comme avantage considérable que ces élèves ont tous derrière eux d'excellents résultats scolaires puisque l'entrée à l'université en Espagne est conditionnée par une note d'entrée ${ }^{6}$ qui varie selon le cursus choisi et qui dans les filières qui nous occupent, est assez élevée. À titre d'exemple, pour avoir accès aux études d'aéronautique, la note minimale demandée pour l'année scolaire 2012-13 est de 12,29 sur 14. De plus, la plupart de ces étudiants a deux langues maternelles, le castillan et le valencien, ce qui facilite considérablement l'apprentissage des langues romanes surtout au niveau des compréhensions orales et écrites. Dans l'enseignement primaire et/ou secondaire, en général, ils ont tous étudié l'anglais. De profil scientifique, ils font partie comme on le sait, des «natifs numériques» (Prenski, 2001) ce que j'interprète comme habitués à manipuler les Technologies de l'information et de la communication (désormais TIC) et surtout aimant le faire.

\subsection{La durée de l'activité}

L'activité est prévue pour une durée de deux heures : une heure pour découvrir les documents et une heure pour la réalisation de l'activité par deux. Pour ce faire, les activités de classe seront bien guidées, les consignes claires, les supports choisis à l'avance par le didacticien, car

6. Appelée «nota de corte». 
ainsi les éparpillements et pertes de temps seront en partie évités. Il convient de rappeler que la gestion du temps est essentielle en français de spécialité. L'activité programmée débouchera obligatoirement sur une production écrite rendue à l'enseignant. Par conséquent, tous les groupes devront avoir effectué la tâche demandée à la fin du cours quelle que soit sa durée.

\subsection{Les supports utilisés}

L'organisation du débat va se faire par le biais des TIC, car elles suscitent la motivation et sont un moyen efficace d'amener les apprenants vers la production, ici écrite. J'utilise d'une part, la plateforme éducative institutionnelle, appelée PoliformaT et d'autre part, le Web.

Parmi les outils de communication (courriels internes, chat, etc.) proposés sur la plateforme, je privilégie le forum. Grâce à cet outil, les étudiants vont pouvoir publier leurs travaux écrits et ces derniers seront donc visibles de tous. Ils pourront aussi être sauvegardés et corrigés.

Sur le Web, le didacticien triera en amont du cours les documents interactifs authentiques sur le sujet à débattre afin de gagner du temps. Pour cette activité, j'ai sélectionné un portail où sont recensés tout type de documents multimodaux dans le but d'activer les mémoires visuelles et auditives et de répondre aux différentes stratégies d'apprentissage des étudiants. Les étudiants sont libres d'y surfer et de choisir les articles et/ou vidéos qui les intéressent le plus et qui, selon eux, leur permettront de répondre aux questions posées. Les documents sonores sélectionnés sont sous-titrés et facilitent la compréhension orale.

\subsection{Le sujet}

Le choix du sujet s'est effectué en tenant compte de l'école d'ingénieur dans laquelle les cours sont dispensés, ici l'école d'ingénieurs en télécommunication : les intérêts des élèves, c'est-à-dire l'importance du thème dans leur vie sociale et les connaissances scientifiques véhiculées. L'objectif recherché est de motiver les apprenants pour qu'ils s'investissent dans la tâche. La problématique proposée, correspondant à une «question socialement vive» (voir section 2 «Bases théoriques»), est celle des risques de la téléphonie mobile pour la santé. 


\subsection{La formation des groupes}

Dans l'intention de niveler les asymétries et de motiver les apprenants dans la réalisation de la tâche, le choix des groupes est effectué par l'enseignant en respectant si possible les intérêts académiques, les liens de camaraderie et en tenant compte de leur niveau de langue française. Ainsi, les niveaux sont mélangés en essayant de former des tandems composés d'un étudiant ayant obtenu de bons résultats avec un de niveau un peu plus faible. Pour cette activité, les étudiants sont par deux et ont à leur disposition un ordinateur par personne et des écouteurs pour pouvoir travailler à leur rythme lors de la découverte des documents et en collaboration, lors de la mise en commun des résultats, l'organisation de l'écrit et la prise de position sur le sujet posé.

\subsection{Les consignes}

Les consignes données sur le forum afin de diriger un écrit argumenté sont les suivantes :
À l'aide d'Internet, cherchez le portail de l'Association nationale pour la sécurité sanitaire dans les technologies sans fil ${ }^{7}$ et sélectionnez des articles de presse, des enquêtes, des interviews ou des vidéos sur le danger des téléphones portables et des antennes relais pour la santé. Répondez aux questions ci-après : Quel est le danger des ondes de type téléphonie mobile? Comment s'en protéger? Êtes-vous pour ou contre leur utilisation? Justifiez votre réponse.

Les étudiants ont aussi à leur disposition une page de dictionnaire bilingue ${ }^{8}$ pour traduire les mots ou les notions qu'ils ne comprennent pas si besoin est, et une page pour vérifier les conjugaisons des verbes et l'orthographe'.

\subsection{Les objectifs pour les apprenants}

L'objectif du débat argumenté est d'abord de rechercher en binôme des informations précises sur la question en cours de débat de manière à enrichir les connaissances scientifiques et langagières des apprenants.

7. (S.d.). Repéré à www.robindestoits.org/

8. Diccionario Espasa Grand: español-francés français-espagnol. (2000). Espasa-Calpe S.A. : Madrid. Repéré à www.wordreference.com/fres/

9. Français langue et culture (s.d.). Repéré à www.orthogram.com/sommaire_ des_fiches_dorthographe_et_de_grammaire.html 
La thèse du débat correspond à la question à résoudre, les arguments aux affirmations soutenant la thèse et les contre-arguments aux allégations en vue de critiquer la thèse principale. Afin de pouvoir répondre par écrit aux questions posées, les étudiants discutent à l'oral les arguments relevés dans les documents virtuels et essaient de trouver un terrain d'entente pour justifier leur utilisation du portable. Finalement, ils doivent encore synchroniser leurs opinions et leurs connaissances linguistiques à l'écrit pour terminer la tâche demandée. Pour ce faire, ils peuvent réutiliser les contenus des unités 6 et 11 vus dans la partie du français général et cités supra.

\section{Résultats et discussion}

Pour montrer que les élèves de niveau élémentaire sont capables d'argumenter, j'ai inventorié les types d'arguments auxquels ils ont eu recours dans leurs écrits en reprenant les grandes classes d'arguments décrites par Leclerc (1999, p. 20-35) pour les discours scientifiques. Celui-ci différencie trois groupes d'arguments : ceux de type scientifique qui font appel aux faits comme preuves, aux données chiffrées, aux témoignages, à l'autorité, aux conclusions d'études; les arguments «intellectualisés», reliés à une opération de l'esprit (la logique, le rapprochement des concepts, etc.), au raisonnement de cause à effet qui «consiste à établir un rapport entre deux faits, évènements ou phénomènes dont le premier (la cause) est présenté comme étant à l'origine de l'autre (l'effet). [...] ce type d'argument peut s'avérer efficace lorsqu'on veut faire partager son point de vue», à la concession, à l'hypothèse et à la crédibilité de l'émetteur. Et finalement, les arguments subjectifs qui se réfèrent à la définition, la tradition, la nouveauté, les sentiments, etc.

Le tableau 1 ci-après expose un échantillon représentatif des arguments utilisés dans les écrits des étudiants élaborés en binômes, et ce, afin d'établir leur typologie.

Les travaux des étudiants mettent en exergue : des arguments scientifiques qui ont recours aux faits, à l'autorité ainsi qu'à des conclusions d'études; des arguments «intellectualisés » parmi lesquels on observe des raisonnements de cause à effet; des concessions avec l'utilisation de la conjonction de coordination «mais» et les conséquences ou solutions à la question posée; enfin les arguments subjectifs (adaptation à la vie moderne, nécessité d'utilisation) qui ont pour fonction de clore le raisonnement et permettent aux étudiants de prendre position. 


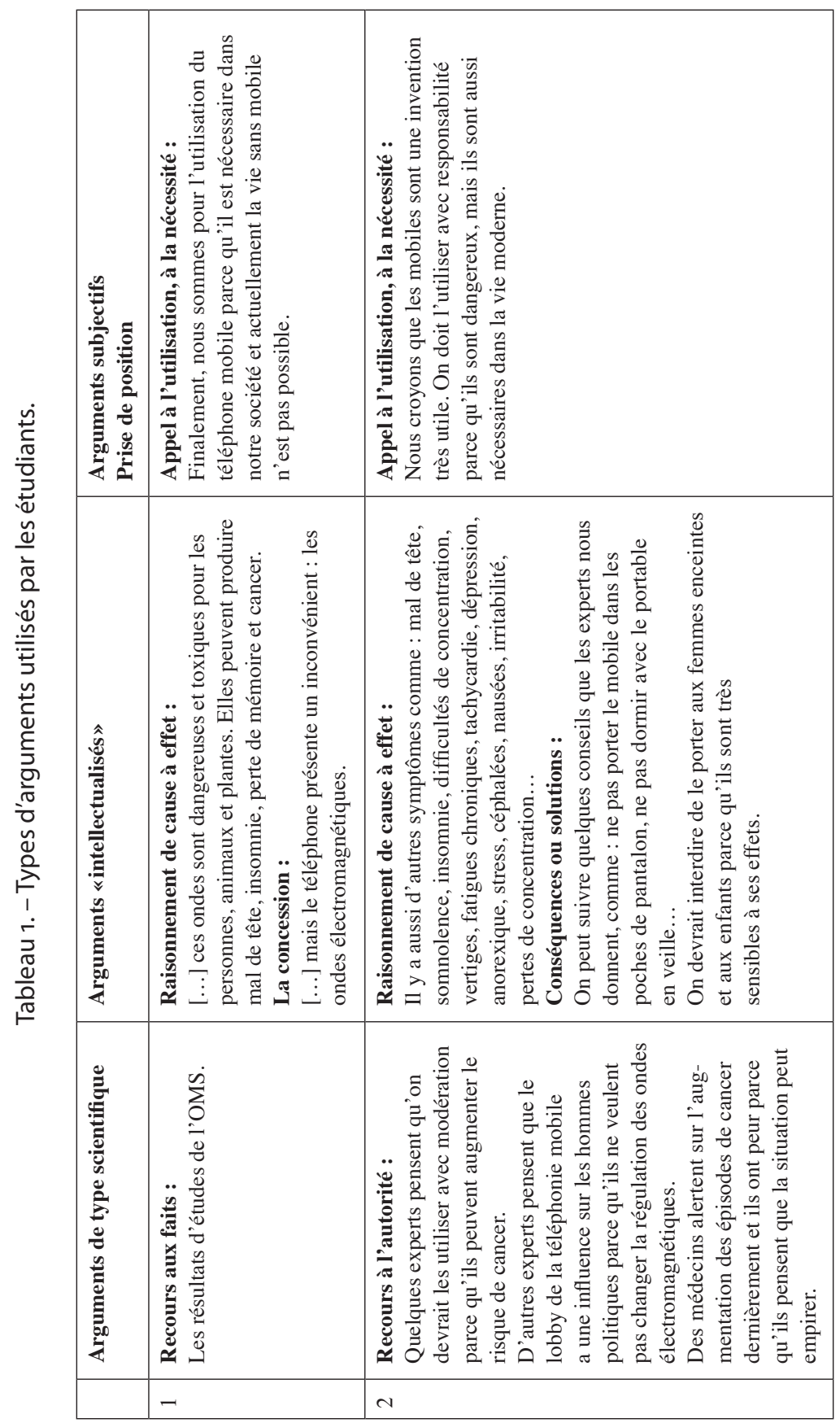




\begin{tabular}{|c|c|}
\hline 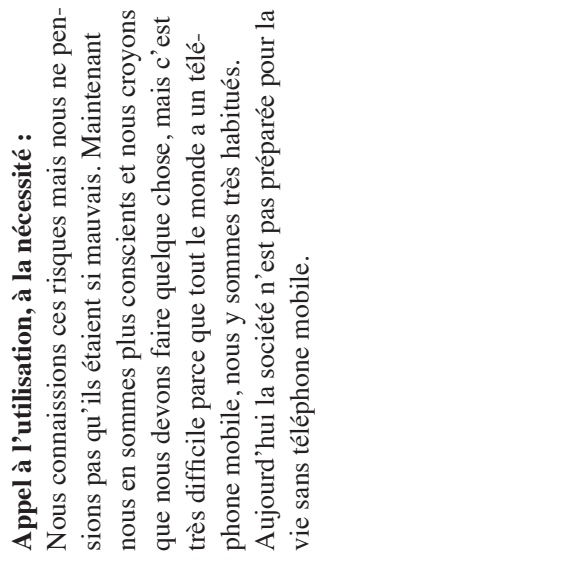 & 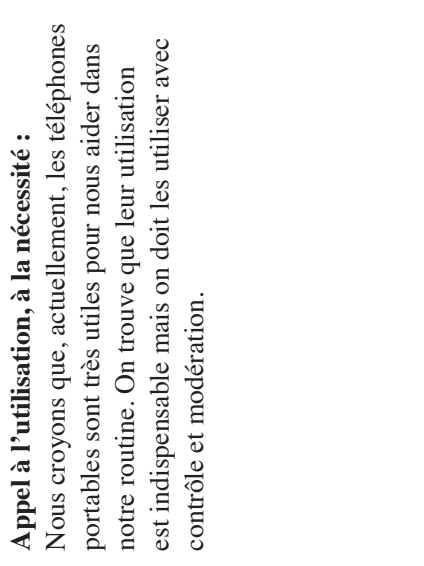 \\
\hline 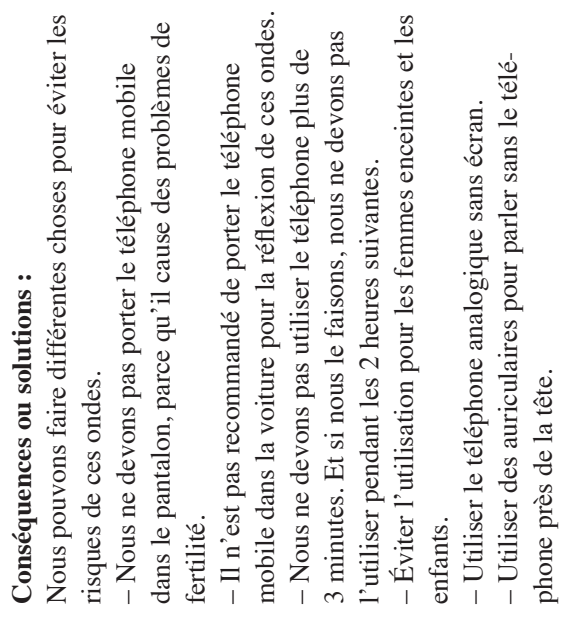 & 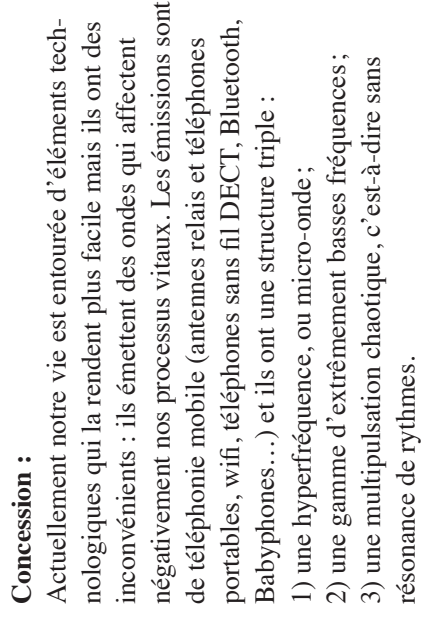 \\
\hline 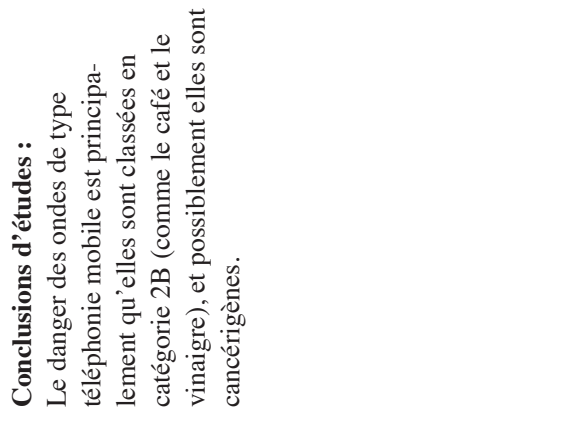 & 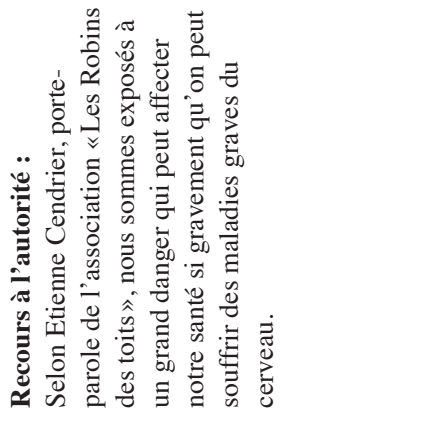 \\
\hline$m$ & $\nabla$ \\
\hline
\end{tabular}




\begin{tabular}{|c|c|}
\hline & 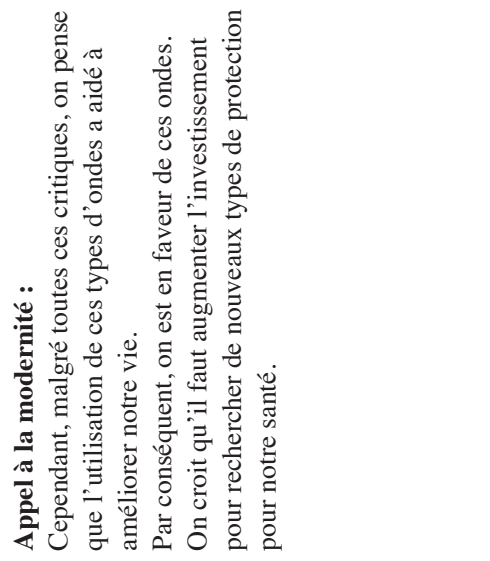 \\
\hline \multirow[t]{3}{*}{ 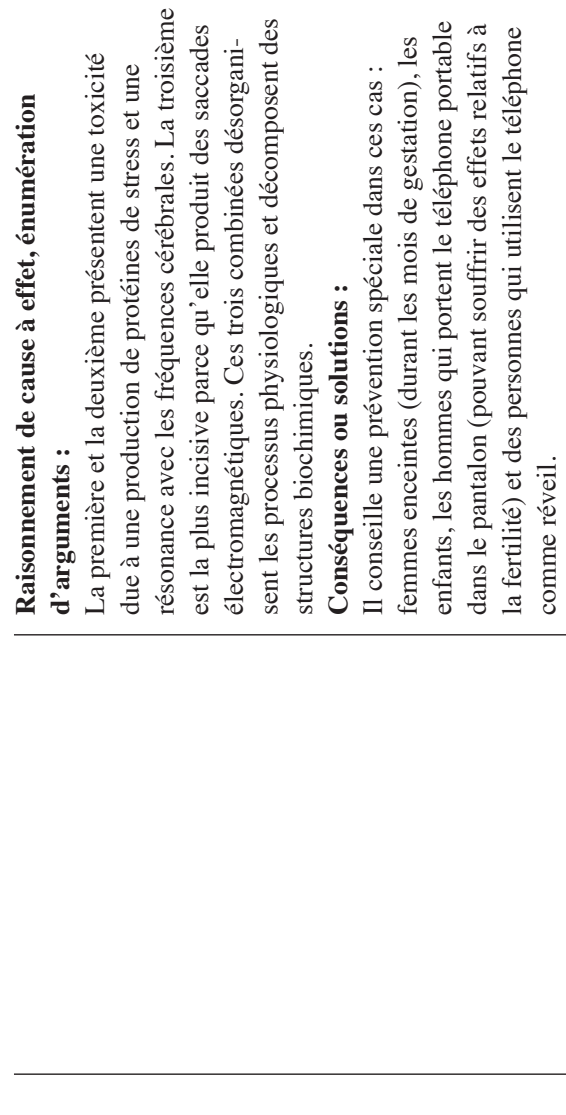 } & 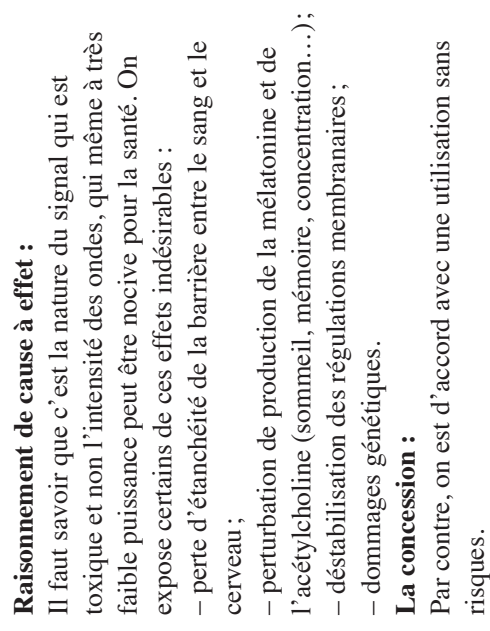 \\
\hline & 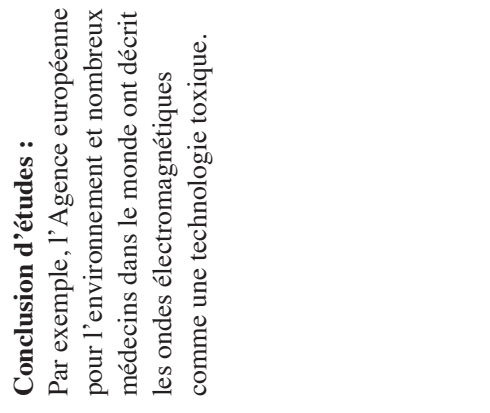 \\
\hline & in \\
\hline
\end{tabular}


Les arguments de type scientifique et intellectualisés utilisés mobilisent ou impliquent des connaissances scientifiques acquises, pour certaines, lors de l'activité. L'accès à un portail français sur les risques de la téléphonie mobile a permis aux étudiants d'actualiser leurs connaissances scientifiques sur le sujet et de manipuler le vocabulaire employé dans ces discours de spécialité. Ainsi, pour expliquer les types d'ondes électromagnétiques, ils ont dû tout d'abord comprendre et identifier les unités lexicales spécialisées simples et complexes du champ de la téléphonie mobile et ensuite les réemployer. On relève à titre d'exemple, dans leurs écrits :

les antennes relais GMS, l'UMTS, les ondes WIFI, le Wimax, les téléphones portables, les téléphones sans fil DECT, le téléphone filaire, Bluetooth, Babyphones, hyperfréquence, micro-onde, une gamme de basses fréquences, une multipulsation chaotique, sans résonance de rythmes, etc.

En outre, le sujet traité leur a permis de travailler aussi la terminologie liée au domaine scientifique de la médecine puisque certaines maladies citées dans les articles correspondent aux conséquences possibles de l'utilisation des portables :

mal de tête, somnolence, insomnie, difficultés de concentration, pertes de mémoire, vertiges, fatigue chronique, tachycardie, dépression, anorexie, stress, céphalées, nausées, irritabilité, cancer, etc.

L'utilisation d'arguments faisant appel à l'autorité de témoignage leur a donné une ouverture à la culture scientifique puisqu'ils ont pu lire ou voir et écouter des études et des rapports scientifiques officiels nationaux et internationaux (comme les rapports $\operatorname{Cost}^{10} 281$, Reflex ${ }^{11}$ sur la 3G de l'Union européenne, etc.); des avis, des commentaires et des analyses scientifiques d'experts, entre autres, les médecins de l'Asef (Association Santé Environnement France); des appels et mises en garde de médecins ou d'experts scientifiques comme le professeur Luc Montagnier, prix Nobel de médecine en 2008. Cet accès au champ culturel des sciences est, à mon avis, indispensable pour développer leur compétence critique, base de leur avenir comme futurs chercheurs.

10. COST: European Cooperation in the Field of Scientific and Technical Research.

11. REFLEX: Risk Evaluation of Potential Environmental Hazards From Low Frequency Electromagnetic Field Exposure Using Sensitive in vitro Methods. 
Néanmoins, pour le sujet choisi, ces types d'arguments ne parviennent pas à modifier totalement les représentations initiales des étudiants. En effet, ce sont les arguments subjectifs liés aux besoins d'usage du portable dans la vie de tous les jours qui l'emportent dans les prises de position en conclusion. Cependant, quelques-uns manifestent leur intention de modifier quelque peu certaines de leurs habitudes. Les apprenants sont bien conscients que la science peut évoluer et suggèrent que des recherches soient entreprises pour éviter toutes les conséquences négatives citées par les chercheurs. Il est évident que malgré leur niveau A2 dans l'apprentissage de la langue française, les étudiants sont arrivés, en s'appuyant sur les documents fournis, à construire un discours argumenté écrit et à expliquer les différentes solutions au problème des ondes électromagnétiques existant actuellement.

\section{Conclusion}

La question socialement vive traitée ici, de par son caractère interdisciplinaire, a permis le décloisonnement de plusieurs domaines de référence, celui des télécommunications, celui de la médecine et celui de la société. Les discours, débats ou arguments apportés au travers des documents par les spécialistes du domaine prouvent qu'effectivement, comme je le signalais dans la partie théorique, même si la langue scientifique rend compte des connaissances du domaine étudié et comporte des unités lexicales spécialisées, elle n'est en aucune façon une autre langue et que pour exister dans la communication, dans les médias, elle a besoin de la langue générale.

Lors de la réalisation de l'activité, les étudiants ont pris contact avec la culture scientifique française (ils ont connu des experts français et internationaux du domaine de la télécommunication et de la médecine, ainsi que des organismes officiels). Ils ont développé la compréhension de l'écrit et de l'oral à partir de documents authentiques, observé le fonctionnement des unités lexicales spécialisées en discours et réemployé les arguments scientifiques pour justifier les réponses aux questions demandées. Ils se sont initiés au débat argumenté : à l'oral en binômes, à l'écrit pour répondre aux questions et ils ont commencé, par là-même, à poser les premiers jalons de leurs futurs discours scientifiques.

Je peux affirmer que les objectifs d'apprentissage ont été atteints, car à travers le débat, les apprenants ont acquis des connaissances scientifiques et linguistiques, le processus d'apprentissage s'est basé sur l'action 
et ils ont argumenté en langue étrangère. Malgré leur niveau élémentaire de français, ils sont arrivés à structurer leurs propos et à employer des arguments que l'on rencontre habituellement dans les discours scientifiques. Je conclus donc que l'argumentation peut être utilisée en fin de niveau A2 en tant qu'un moyen pour faire progresser les connaissances et développer la compétence de communication langagière. Cependant, un cadrage minutieux des activités est nécessaire pour réussir ce type de tâche à ce niveau élémentaire.

\section{RÉFÉRENCES BIBLIOGRAPHIQUES}

Albe, V. (2006). Procédés discursifs et rôles sociaux d'élèves en groupes de discussion sur une controverse socio-scientifique. Revue française de pédagogie, 157, 103-118.

Ausubel, D. (1968). Educational psychology: a cognitive view. New York: Holt, Rinehart and Winston.

Bédard, C. (1986). La traduction technique. Principes et pratique. Montréal : Linguatech.

Bruner, J. (1960). The Process of Education. Cambridge, MA : Havard University Press.

Buty, C. \& Plantin, C. (2008). Argumenter en classe de sciences. Du débat à l'apprentissage. Lyon : INRP.

Cabré, M.-T. (2007-2008). Constituer un corpus de textes de spécialité. Cahiers du CIEL, 37-56.

Commission GÉnérale de terminologie et de nÉOlogie. (2000). Grand dictionnaire terminologique. Office québécois de la langue française. Repéré à http://gdt.oqlf.gouv.qc.ca/ficheOqlf.aspx?Id_Fiche=111807

Conseil de L'Europe. (2001). Cadre européen commun de référence pour les langues. Paris : Didier.

Collectif DE RECHERCHE EXPÉRIMENTALE ENSEIGNEMENT FRANÇAIS (CRESEF). (1997). Enseigner 1'argumentation, Pratiques, 96.

CuQ, J.-P. (dir.). (2003). Dictionnaire de didactique du français. Paris : CLE International/Asdifle.

Dolz, J. \& Schneuwly, B. (1998). Pour un enseignement de l'oral : initiation aux genres formels à l'école. Paris : ESF Éditeur.

DouAire, J. (dir.). (2004). Argumentation et disciplines scolaires. Lyon : INRP.

Genelot, D. (2011). Sur l'exercice de la pensée complexe. L'éditorial du Réseau Intelligence de la Complexité. Associations AE-MCX et APC. Repéré à http://archive.mcxapc.org/docs/1102pres22mars.pdf 
Golder, C. (1996). Le développement des discours argumentatifs. Lausanne, Paris : Delachaux et Niestlé.

Grize, J.-B. (1990). Logique et Langage. Paris et Gap : Ophrys.

Jacobi, D. \& Peterfalvi, B . (2004). Les interactions langagières 2. Aster, 38. Saint-Fons, France : INRP-ASTER.

Joshua, S. \& Dupin, J.-J. (1989). Représentations et modélisations : le «débat scientifique» dans la classe et l'apprentissage de la physique. Berne : Peter Lang.

Kocourek, R. (1991). La langue française de la technique et de la science : vers une linguistique de la langue savante ( $2^{\mathrm{e}}$ édition). Wiesbaden : O. Branderstetter.

LECLERC, J. (1999). Le français scientifique : guide de rédaction et de vulgarisation. Brossard : Linguatech Éditeur.

Legardez, A. et Simmoneaux, L. (2006). L'école à l'épreuve de l'actualité. Enseigner les questions vives. Issy-les-Moulineaux : ESF.

Mangiante, J.-M. et Parpette, C. (2004). Le Français sur Objectif Spécifique : de l'analyse des besoins à l'élaboration d'un cours. Paris : Hachette FLE.

Morin, E. (mise en ligne en février 2003). Articuler les disciplines. Forum scientifique du programme européen. Associations AE-MCX et APC. Repéré à http://archive.mcxapc.org/docs/conseilscient/morin1.htm

Olmo, F. (2008). Quelle méthodologie adopter pour des élèves débutants en Français sur Objectifs Spécifiques? Dans O. Bertrand et I. Schaffner (dir.), Le français de spécialité. Enjeux culturels et linguistiques (p. 197-206). Palaiseau : Éditions de l'École polytechnique.

Plantin, C. (1996). L'argumentation. Paris : Seuil.

Prenski, M. (2001). Digital Natives, Digital Immigrants. On the Horizon, 9 (5), 1-6. Repéré à www.marcprensky.com/writing/prensky - digital natives, digital immigrants - part1.pdf

Simmoneaux, L. \& Simmoneaux, J. (2005). Argumentation sur des questions socio-scientifiques. Didaskalia, 27, 79-108.

Simmoneaux, L. et Albe, V. (2008). Types et domaines d'arguments utilisés dans des débats socio-scientifiques. Dans C. Buty et C. Plantin (dir.), Argumenter en classe de sciences. Du débat à l'apprentissage (p. 117191). Lyon : INRP.

Vygotsky, L. (1978). Interaction between learning and development. Cambridge, MA : Havard University Press.

Widdowson, H. (1991). Une approche communicative de l'enseignement des langues. LAL. Paris : Didier/Hatier. 